\title{
Monitoramento e avaliação da segurança alimentar e nutricional: um olhar sobre as publicações oficiais
}

\author{
Monitoring and evaluation of food and nutritional security: \\ a look at the official publications
}

Amanda da Silva Bastos de Oliveira (https://orcid.org/0000-0001-9963-8191) ${ }^{1}$ Juliana Pereira Casemiro (https://orcid.org/0000-0001-6940-2479) ${ }^{2}$

Ana Laura Brandão (https://orcid.org/0000-0002-7148-2268) ${ }^{1}$

Alessandra Maria Silva Pinto (https://orcid.org/0000-0002-0814-125X) ${ }^{3}$
${ }^{1}$ Escola Nacional de Saúde Pública Sergio Arouca, Fundação Oswaldo Cruz. R. Leopoldo Bulhões 1.480, Manguinhos. 21041-210 Rio de Janeiro RJ Brasil. amandaoliveiranut@ gmail.com

${ }^{2}$ Instituto de Nutrição, Universidade do Estado do Rio de Janeiro. Rio de Janeiro RJ Brasil.

${ }^{3}$ Instituto Brasileiro de

Geografia e Estatística. Rio de Janeiro RJ Brasil.
Abstract Food and Nutrition Security, the legal frameworks to guarantee and enforce the Human Right to Adequate Food and construction of a system to monitor and evaluate progress and setbacks in these processes are the outcome of collective efforts led by social movements, organized civil society and some areas of government. This article examines official Brazilian documents regarding such monitoring and evaluation published from 2003 to 2019. The documentary analysis and contextualization were framed by the dimensions specified in Decree No. 7272/2010. The analysis highlighted the importance of the National Food and Nutrition Security Council (CONSEA), the Interministerial Food and Nutrition Security Chamber (CAISAN) and the Brazilian Food and Nutrition Security Conferences in the construction of monitoring and evaluation mechanisms. The challenges identified in this process stem from inter-sector relations and decentralization of actions, which tend to be weakened in certain political contexts.

Key words Food and nutrition security, Program evaluation, Monitoring, Inter-sector collaboration
Resumo A segurança alimentar e nutricional (SAN), os marcos legais para a garantia e exigibilidade do direito humano à alimentação adequada (DHAA) e a construção de um sistema que monitore e avalie os avanços e retrocessos nesses processos são provenientes dos esforços coletivos impulsionados por movimentos sociais, sociedade civil organizada e alguns segmentos governamentais. Este artigo analisa documentos oficiais produzidos nacionalmente e publicados entre $2003 e$ 2019 na perspectiva de monitoramento e avaliação $(M \triangleleft A)$, com a realização de análise documental e contextualização fundamentada nas dimensões do Decreto $n^{\circ} 7.272 / 2010$. Como resultado, destaca-se a importância do Conselho Nacional de Segurança Alimentar e Nutricional, da Câmara Interministerial de Segurança Alimentar e Nutricional e das Conferências Nacionais de SAN na construção de mecanismos de MઐA. Os desafios destacados nesse processo se relacionam com as articulações intersetoriais e a descentralização das ações, que tendem a se fragilizar, dependendo dos contextos políticos.

Palavras-chave Segurança alimentar e nutricional, Avaliação de programas e projetos de saúde, Monitoramento, Colaboração intersetorial 


\section{Introdução}

A trajetória da Política Nacional de Segurança Alimentar e Nutricional (PNSAN) é fruto de amplo processo de participação popular e de articulação de esforços de governo, academia e organizações populares. Germinada a partir do processo de redemocratização, iniciado na década de 1980, a defesa da segurança alimentar e nutricional (SAN) se desdobra da mobilização pela reforma sanitária e pela ética na política, das campanhas de combate à fome e de outras expressões de organização de vínculo e solidariedade. Em 2003, as ações desse campo foram impulsionadas mediante estratégia do governo federal e buscaram se concretizar nos demais entes federativos a partir do Sistema Nacional de Segurança Alimentar e Nutricional (SISAN) ${ }^{1}$.

O SISAN se propõe a efetivar o direito humano à alimentação adequada (DHAA) e a SAN da população brasileira, a partir da integração de diversos órgãos, entes federativos e instituições privadas - com ou sem fins lucrativos -, bem como de critérios estabelecidos pelo Conselho Nacional de Segurança Alimentar e Nutricional (CONSEA) e pela Câmara Interministerial de Segurança Alimentar e Nutricional (CAISAN). Constam, entre seus objetivos, "formular e implementar políticas e planos de SAN, estimular a integração dos esforços entre governo e sociedade civil, além de promover o acompanhamento, o monitoramento e a avaliação da SAN no país" (p. 1) $)^{2}$. Portanto, o monitoramento e a avaliação (M\&A) em SAN constituem uma das diretrizes estabelecidas para o SISAN ${ }^{2}$.

Entre as diversas definições do processo de avaliação, existe um consenso quanto à formulação de julgamento de valor a respeito de uma intervenção, a depender dos atores envolvidos nessa dinâmica em tempos estipulados e de critérios determinados ${ }^{3,4}$. Em relação ao monitoramento, entende-se que é realizado continuamente com a finalidade de fornecer informações e mensurar avanços e/ou retrocessos em relação aos resultados. M\&A são essenciais para a tomada de decisão, seja no âmbito do poder público ou da sociedade civil ${ }^{4}$.

Coerente com a ampla contribuição da sociedade civil na construção do campo e da PNSAN, o Decreto $n^{\circ} 7.272 / 2010$ propõe a efetivação de um sistema de M\&A pautado em participação, transparência, publicidade, facilidade de acesso à informação e atento à implementação da Política, bem como dos objetivos e metas estabelecidas nos Planos Nacionais de Segurança Alimentar e
Nutricional (PLANSAN). O uso de indicadores dos diversos setores relacionados à SAN é destacado no decreto como base do sistema de $M \& \mathrm{~A}^{5}$, inclusive aqueles que expressam desigualdades sociais, de gênero e étnico-raciais.

O presente artigo tem como objetivo analisar as propostas para M\&A expostas em documentos produzidos pelo CONSEA e pela CAISAN publicados de 2003 a 2019 e correlacioná-los com os contextos políticos inerentes ao período.

\section{Métodos}

A pesquisa documental foi realizada em outubro e novembro de 2019. O reconhecimento do CONSEA e da CAISAN como "fontes potenciais de informação"6 sobre M\&A em SAN definiu a opção metodológica de análise de sua produção.

Entre os documentos de domínio público encontrados, publicados entre 2003 e 2019, constam 149 Exposições de Motivos $^{7}$ (EM) e 43 publicações presentes na Central de Conteúdo do CONSEA, além de 19 da CAISAN, totalizando 211 publicações. Desse total foram selecionadas 13 publicações que continham as seguintes expressões de busca: "avaliação", "monitoramento", "avaliar" ou "monitorar".

Todo o materiat selecionado foi, posteriormente, organizado em planilha do Microsoft Excel de acordo com nome, órgão, ano da publicação e conteúdo, e sistematizado mediante "leitura flutuante" com base nas dimensões de análise descritas no Decreto no 7.272/2010, a saber: I -Produção de alimentos; II - Disponibilidade de alimentos; III - Renda e condições de vida; IV - Acesso à alimentação adequada e saudável, incluindo água; $\mathrm{V}$ - Saúde, nutrição e acesso a serviços relacionados; VI - Educação; e VII - Programas e ações relacionadas à segurança alimentar e nutricional.

A análise documental empregou metodologia proposta por Cellard ${ }^{6}$, utilizando cinco dimensões preliminares: contexto da produção do documento, abarcando as considerações do autor e demais envolvidos; observação de autor(es), bem como os posicionamentos e as motivações; qualidade das informações e relação do(s) autor(es) com o documento; atenção ao modo com que as informações são transmitidas; e delineamento dos sentidos expressos, analisando os conceitos que aparecem e a importância deles. A análise documental, em seu sentido amplo, foi empregada após todos esses passos terem sido executados. 


\section{Monitoramento e avaliação na legislação brasileira de SAN}

Figueiró, Frias e Navarro ${ }^{9}$ resumem os objetivos principais da avaliação em: (1) auxiliar no planejamento e desenvolvimento de uma intervenção (objetivo estratégico); (2) subsidiar, por meio de informações, a melhoria de uma intervenção durante sua implementação (objetivo formativo); (3) determinar efeitos de uma intervenção para decidir sua manutenção, alteração ou interrupção (objetivo somativo); (4) utilizar processos de avaliação como incentivo à transformação de situações injustas ou problemáticas, visando o bem-estar (objetivo transformador); ou (5) contribuir para o progresso dos conhecimentos e para a elaboração teórica (objetivo fundamental).

O CONSEA e a CAISAN, em conjunto, exerceram atribuições importantes no M\&A da SAN. O primeiro CONSEA foi instituído em 1993 como demanda do Movimento Ética pela Política e atuou até 1995, quando foi extinto e substituído pelo Programa Comunidade Solidária ${ }^{10-12}$.

Em 2003, o CONSEA foi reinstituído, exercendo papel fundamental para o diálogo entre sociedade e governo, com a seguinte composição: dois terços de representantes da sociedade civil e um terço governamental, sendo a presidência do conselho exercida por um membro da sociedade civil $^{10,12,13}$. A criação da CAISAN, em 2007, composta por representantes ministeriais, propiciou a articulação das ações de SAN com órgãos federais mediante interlocução com o CONSEA ${ }^{14}$.

Essa atuação contígua foi exercida até janeiro de 2019, quando o CONSEA foi extinto pela segunda vez por meio da Medida Provisória no 870, com posterior veto à emenda apresentada pela sociedade civil organizada e parlamentares ${ }^{15,16}$. Quando vigente, o CONSEA foi responsável por articular, acompanhar, mobilizar e monitorar ações propostas na política e nos planos com os demais órgãos e entidades do SISAN. A CAISAN é responsável pela elaboração da política, dos planos, instrumentos avaliativos e de monitoramento, inclusive na coordenação da articulação com congêneres estaduais e do Distrito Federal ${ }^{2}$.

As Conferências Nacionais de Segurança Alimentar e Nutricional (CNSAN), instâncias máximas de deliberação, influenciaram no desenvolvimento de políticas públicas, propondo à CAISAN diretrizes que compuseram a Política e os PLANSAN. As conferências ocorreram em cinco edições e, nos intervalos, foram realizados os Encontros Conferências +2 , tendo como ob- jetivo realizar balanços das proposições das conferências ${ }^{2,17}$.

Destacam-se, entre os legados desse período, a produção e consolidação de um campo teórico e político relacionado ao M\&A em SAN e DHAA. Esse caminho pode ser reconstituído a partir de diferentes documentos, sobretudo no período entre a criação do SISAN, pela Lei Orgânica de Segurança Alimentar e Nutricional LOSAN, até a sua regulamentação, via Decreto $n^{\circ}$ $7272 / 2010^{2,5}$.

No âmbito do governo federal, foi criada, em 2004, a Secretaria de Avaliação e Gestão da Informação (SAGI), assumindo atribuições de monitoramento e avaliação de políticas e programas do Ministério do Desenvolvimento Social. Sua metodologia foi classificada como uma importante referência em pesquisas de avaliação ${ }^{18,19}$.

Dando centralidade à temática de M\&A, o CONSEA criou, em 2006, o Grupo Técnico Indicadores e Monitoramento, que se reuniu de forma sistemática até 2010. A culminância de seu trabalho se deu com a publicação do relatório "A segurança alimentar e nutricional e o direito humano à alimentação adequada", que orientou e inspirou a definição das dimensões de monitoramento, descritas no Decreto no $7272 / 2010^{20,21}$.

Observou-se, após a publicação do decreto, o aumento do número de publicações sobre M\&A, tanto de caráter normativo quanto de organização de indicadores e mapeamento da SAN, nas páginas da CAISAN e do CONSEA. No sítio da CAISAN, dos 19 documentos encontrados, cinco tratam do tema. Das 192 publicações do CONSEA, oito tratam de monitoramento e/ou avaliação. O Quadro 1 exibe os documentos segundo órgão, entidade de expedição e ano de publicação.

$\mathrm{Na}$ leitura do material, buscou-se analisar a perspectiva de M\&A expressa e sua relação com a conjuntura política da SAN. Observa-se um percurso iniciado pela afirmação do M\&A enquanto parte integrante do arcabouço legal do SISAN e como elemento central do controle social frente às obrigações do Estado com relação ao $\mathrm{DHAA}^{22,23}$. Essa concepção é reforçada pela incorporação do conceito de exigibilidade de direitos nas publicações do CONSEA ${ }^{20,24,25}$, e fruto da aprovação da Emenda Constitucional no $64^{26}$, que inclui a alimentação entre os direitos sociais e reafirma as competências do Estado brasileiro.

O passo seguinte na configuração do M\&A, em âmbito nacional, foi a produção de subsídios para estados e municípios ${ }^{27}$. A movimentação de descentralização da gestão do SISAN propôs 
Quadro 1. Documentos oficiais publicados segundo órgão/entidade de expedição e ano de publicação.

\begin{tabular}{|c|c|c|}
\hline Documento & Órgão/entidade & $\begin{array}{c}\text { Ano da } \\
\text { publicação }\end{array}$ \\
\hline $\begin{array}{l}\text { Construção do Sistema e da Política Nacional de } \\
\text { Segurança Alimentar e Nutricional: a experiência } \\
\text { brasileira }\end{array}$ & CONSEA, FAO, IICA & 2009 \\
\hline $\begin{array}{l}\text { Avanços e desafios na implementação do direito } \\
\text { humano à alimentação adequada }\end{array}$ & CERESAN, ABRANDH, CONSEA & 2009 \\
\hline $\begin{array}{l}\text { A segurança alimentar e nutricional e o direito humano } \\
\text { à alimentação adequada - indicadores e monitoramento } \\
\text { da Constituição de } 1988 \text { aos dias atuais }\end{array}$ & CONSEA & 2010 \\
\hline $\begin{array}{l}\text { A exigibilidade do direito humano à alimentação } \\
\text { adequada e o Sistema Nacional de Segurança Alimentar } \\
\text { e Nutricional - relatório final }\end{array}$ & $\begin{array}{l}\text { FAO, ABRANDH, CONSEA, } \\
\text { FOME ZERO, Ministério do } \\
\text { Desenvolvimento e Combate à Fome, } \\
\text { Governo Federal }\end{array}$ & 2010 \\
\hline $\begin{array}{l}\text { Estruturando o Sistema Nacional de Segurança } \\
\text { Alimentar e Nutricional }\end{array}$ & CAISAN & 2011 \\
\hline $\begin{array}{l}\text { Agroecologia e o direito humano à alimentação } \\
\text { adequada }\end{array}$ & CAISAN & 2012 \\
\hline $\begin{array}{l}\text { Volatilidade dos preços internacionais e inflação } \\
\text { de alimentos no Brasil: fatores determinantes e } \\
\text { repercussões na segurança alimentar e nutricional }\end{array}$ & CAISAN & 2013 \\
\hline $\begin{array}{l}\text { Estratégia intersetorial de prevenção e controle da } \\
\text { obesidade - recomendações para estados e municípios }\end{array}$ & $\begin{array}{l}\text { CAISAN e Ministério do } \\
\text { Desenvolvimento Social e Combate à } \\
\text { Fome (MDS) }\end{array}$ & 2014 \\
\hline $\begin{array}{l}\text { Estudo técnico - mapeamento da insegurança alimentar } \\
\text { e nutricional com foco na desnutrição a partir da análise } \\
\text { do cadastro único e do Sisvan }\end{array}$ & CAISAN & 2014 \\
\hline $\begin{array}{l}\text { MAPASAN - Mapeamento da Segurança Alimentar e } \\
\text { Nutricional }\end{array}$ & $\begin{array}{l}\text { MDS, Secretaria de Avaliação e } \\
\text { Gestão da Informação (SAGI/MDS), } \\
\text { Secretaria Nacional de Segurança } \\
\text { Alimentar e Nutricional, CAISAN }\end{array}$ & 2015 \\
\hline $\begin{array}{l}\text { A exigibilidade do direito humano à alimentação } \\
\text { adequada - ampliando a democracia no SISAN }\end{array}$ & CAISAN & 2016 \\
\hline $\begin{array}{l}\text { Compromissos do Brasil para a Década de Ação das } \\
\text { Nações Unidas para a Nutrição (2016-2025) }\end{array}$ & $\begin{array}{l}\text { CAISAN e Ministérios e Secretarias } \\
\text { Especiais e Nacionais }\end{array}$ & 2016 \\
\hline $\begin{array}{l}\text { Estratégia intersetorial para a redução de perdas e } \\
\text { desperdício de alimentos no Brasil }\end{array}$ & CAISAN & 2018 \\
\hline
\end{tabular}

Fonte: Autores.

a promoção da autonomia dos entes federativos, bem como sua articulação coordenada. Esses pilares ainda hoje constituem desafios, expressos nas dificuldades de focalização em programas específicos, nas limitadas articulações existentes e no fraco poder de estímulo do governo federal especialmente em se tratando de municípios ${ }^{28,29}$.

Vale ressaltar a instituição de mapeamentos pela CAISAN entre 2014 e 2016: os MAPASANs tiveram como objetivo avaliar continuamente a gestão local da PNSAN por meio de indicadores de adesão dos estados e municípios ao SISAN. Já o MAPAINSAN registrou especificamente a situação de SAN, buscando identificar áreas onde ocorrem violações do DHAA, com dados do Cadastro Único, do SISVAN e do Sistema de Informação da Atenção à Saúde Indígena (SIA$\mathrm{SI})$. Os panoramas oferecidos contribuíram para o monitoramento da PNSAN e das ações locais, e podem orientar a formulação de políticas públicas $^{30,31}$.

As reflexões sobre o modelo de produção impactaram os documentos, pautando questões relacionadas à contribuição da agroecologia ${ }^{32} \mathrm{e}$ da agricultura familiar para o abastecimento alimentar brasileiro, além de outros aspectos rela- 
cionados a produção, disponibilidade e preço de alimentos ${ }^{33}$. Da mesma maneira, as mudanças no perfil epidemiológico da população, sobretudo relacionadas ao aumento da obesidade, influenciaram especialmente o monitoramento de ações de educação alimentar e nutricional e de vigilância alimentar e nutricional ${ }^{34}$. Em todos esses casos, foram observadas manifestações favoráveis à desagregação dos dados para permitir um olhar mais apurado para a população em suas diferenças e desigualdades.

No que se refere ao debate internacional, destaca-se que a agenda do CONSEA-CAISAN promoveu reflexão alinhada aos Objetivos do Desenvolvimento Sustentável (ODS)/Agenda 2030, abarcando metas globais de alimentação e nutrição, tais como prevenção de doenças crônicas não transmissíveis, regulamentação de alimentos industrializados ${ }^{35}$, redução de perdas e combate ao desperdício ${ }^{36}$.

Em linhas gerais, os documentos publicados pelo CONSEA explicitam conteúdos majoritariamente voltados para a intersetorialidade, com estímulo a ações e sistemas descentralizados. O desafio da articulação e implementação de políticas públicas mostra-se bastante expressivo, principalmente em nível municipal, mesmo com a publicação de diversos documentos orientado$\mathrm{res}^{28}$. Já os documentos publicados pela CAISAN dizem respeito à estruturação do sistema, bem como com à aplicabilidade do disposto nas legislações de SAN, com destaque para as dimensões de monitoramento estabelecidas no Decreto $\mathrm{n}^{\circ}$ $7272 / 2010$.

Poucos documentos, tanto da CAISAN como do CONSEA, trataram exclusivamente de M\&A. Observou-se que a CAISAN produziu maior número de documentos de autoria exclusiva, ao contrário do CONSEA, cujos documentos encontrados foram, em maior número, produzidos de maneira conjunta com outras instituições. Cabe ressaltar o importante trabalho desses órgãos tanto no acompanhamento das decisões em âmbito federal como no desenvolvimento de ações e proposições.

\section{Análise das dimensões de avaliação e monitoramento nas Exposições de Motivos do CONSEA}

A partir da atuação do CONSEA, os diálogos entre sociedade civil e governo federal assumiram papel estratégico na inserção da agenda de SAN e das pautas de alimentação e nutrição nos projetos e na construção de políticas públicas.
Essas eram focadas a princípio no enfrentamento da fome, ou seja, na dimensão alimentar. Gradativamente, incorporaram a dimensão nutricional e trouxeram desafios maiores para sua consolidação, dada a necessidade de articulações intersetoriais ${ }^{1}$.

As Exposições de Motivos (EM) constituíram um dos canais de expressão da sociedade civil que o CONSEA utilizava para apresentar as decisões colegiadas sobre temas emergentes de cada período, realizar proposições, impulsionar implementações e demarcar lacunas de políticas públicas. Esses documentos eram destinados à Presidência da República, à CAISAN, aos ministérios e demais órgãos pertinentes às pautas de $\mathrm{SAN}^{7}$.

Foram encontradas 149 EM publicadas no site do CONSEA, 30 delas relacionadas a M\&A. A correlação desse conteúdo com as dimensões do Decreto no 7272/2010, descritas no Quadro 2, resultou na classificação de algumas EM em mais de uma dimensão, principalmente por conta da ampla abrangência da SAN ${ }^{2,37}$.

Houve predomínio de EM classificadas nas dimensões I e VII (Produção de alimentos e Programas e ações relacionadas à $S A N)$. Em estudo sobre a participação do CONSEA no orçamento da União, Oliveira ${ }^{38}$ identificou que mais da metade $(59,7 \%)$ das EM analisadas no período de 2003 a 2013 estava diretamente relacionada à agenda de SAN. Cabe destacar que a análise de EM publicadas entre 2003 e 2019 mostrou-se sensível às mudanças de mandatos presidenciais e do CONSEA, conforme explicitado no Quadro 3.

O início das publicações, em 2003, marcou a retomada do CONSEA no assessoramento à Presidência, dialogando com a agenda prioritária de governo no enfrentamento da fome e da

Quadro 2. Distribuição de exposições de motivos (EM) relacionadas a M\&A publicadas entre 2003 e 2019, segundo dimensões do Decreto 7.272/2010.

\begin{tabular}{|l|c|}
\hline \multicolumn{1}{|c|}{ Dimensão do Decreto 7.272/2010 } & EM \\
\hline I - Produção de alimentos & 11 \\
\hline II - Disponibilidade de alimentos & 8 \\
\hline III - Renda e condições de vida & 2 \\
\hline $\begin{array}{l}\text { IV - Acesso à alimentação adequada e } \\
\text { saudável, incluindo água }\end{array}$ & 6 \\
\hline $\begin{array}{l}\text { V - Saúde, nutrição e acesso a serviços } \\
\text { relacionados }\end{array}$ & 6 \\
\hline VI - Educação & 1 \\
\hline VII - Programas e ações relacionadas à SAN & 22 \\
\hline
\end{tabular}

Fonte: Autores. 


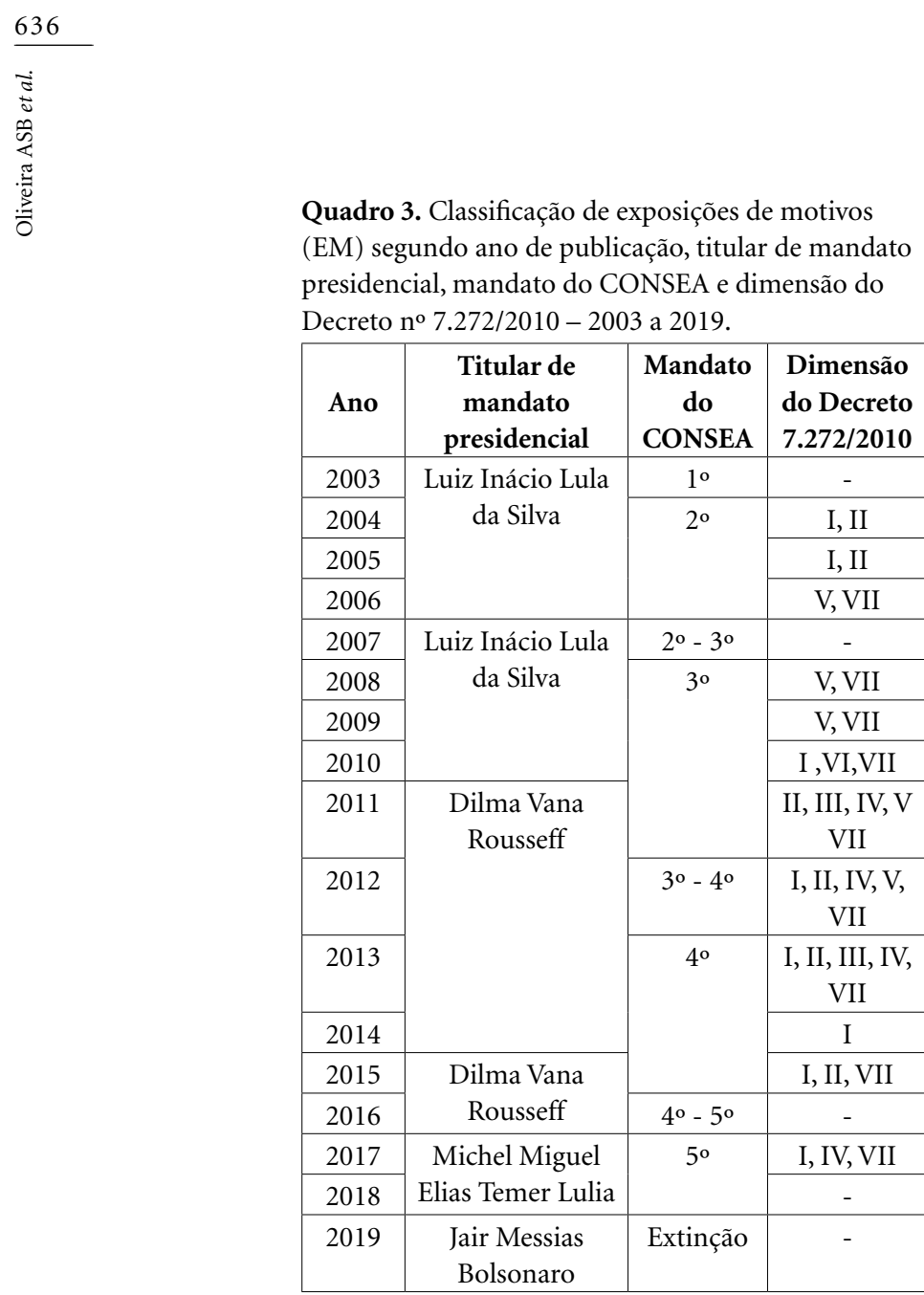

Fonte: Autores.

miséria, por meio de programas como o Bolsa Família (PBF) e o Fome Zero. Essa agenda conferiu centralidade ao tema da SAN, cujas ações já vinham se delineando pelos movimentos sociais e pela sociedade civil desde a redemocratização do país ${ }^{39,40}$.

As EM relativas a M\&A começaram a ser publicadas a partir de 2004. Elas destacavam as dimensões I e II (Produção de alimentos e Disponibilidade de alimentos) até 2005, mostrando a necessidade de avaliação do Plano Safra e do Plano de Aquisição de Alimentos (PAA), bem como a não realização do Censo Agropecuário, em período de incentivos à agricultura familiar $\mathrm{e}$ à reforma agrária na II CNSAN ${ }^{41}$.

A agricultura familiar e a reforma agrária foram amplamente discutidas nas conferências e explicitadas nas EM, pois mesmo com a instituição de programas de valorização de um modelo de produção sustentável havia uma movimentação governamental contraditória de incentivo à produção e exportação de commodities $^{32}$. Em conjunto com essa dimensão, destaca-se o início do delineamento do SISAN com vistas à instituição da PNSAN ${ }^{42}$.

No período de 2006 a 2009, marcado pela implementação da LOSAN em 2006, as dimensões V e VII (Saúde, nutrição e acesso a serviços relacionados e Programas e ações relacionadas à SAN), nas EM, destacaram positivamente o orçamento destinado a programas como o PBF, o PAA e o Programa Nacional de Alimentação Escolar (PNAE). No entanto, um destaque negativo foram as ações de monitoramento da situação nutricional da população: embora tenha ocorrido avanços orçamentários para programas intersetoriais, observou-se limitações institucionais nas ações estruturantes do SISAN, que permaneceram aquém do proposto orçado pelo CONSEA ${ }^{38}$.

A partir de 2010, observou-se a presença de outras dimensões até então ausentes nas EM, como a VI (educação), abordando conquistas relativas ao controle social por meio dos Conselhos de Alimentação Escolar (CAE) e à mobilização de recursos financeiros do PNAE para aquisição de gêneros alimentícios da agricultura familiar e do empreendedor familiar rural ${ }^{43}$. Com relação à produção de alimentos, além da articulação com a educação, observa-se o início dos processos propositivos e de fiscalização em quanto ao uso de agrotóxicos e transgenia, com a sugestão de monitoramento da qualidade dos alimentos e o fortalecimento do Programa Nacional de Análise de Resíduos de Agrotóxicos (PARA).

Entre 2011 e 2013, as EM contemplaram maior número de dimensões que em anos anteriores. Nesse período, a promulgação do Decreto $n^{\circ} 7.272 / 2010$, a realização da IV CNSAN, a elaboração do I PLANSAN e a transição de mandato presidencial - pautado na meta prioritária de erradicação da extrema pobreza por meio do Plano Brasil sem Miséria - foram marcos importantes no campo da SAN. Nessa transição, o teor das EM voltou-se para os preços dos alimentos, a reivindicação de monitoramento dos estoques privados, o modelo de produção e consumo de alimentos, reforçando a necessidade de fortalecer políticas de agricultura familiar, temas que integravam os eixos estratégicos do plano de governo da época ${ }^{44,45}$.

A partir de reivindicações contra o aumento do preço dos alimentos, o CONSEA propôs a criação de uma comissão especial junto à Secretaria Especial de Direitos Humanos, com o objetivo de monitorar possíveis violações do DHAA e instituir mecanismos de exigibilidade desse direi- 
to. Nesse mesmo período, foi instituído o DATASAN, com vistas a monitorar os determinantes da SAN e composto por indicadores alinhados com as dimensões de análise definidas no Decreto $\mathrm{n}^{\circ} 7.272 / 2010^{46}$.

As ações do CONSEA relativas ao crescente incentivo à produção de alimentos por parte do governo federal se pautavam, naquele momento, no M\&A dos impactos do uso de agrotóxicos e da produção de transgênicos, na SAN e no DHAA. Nesse contexto, foram organizadas "mesas de controvérsias" para debater e elaboração de recomendações, destacadas nas EM de 2013 e $2014^{47,48}$. Em 2015, as EM demonstraram a continuidade dessas discussões e propuseram programas de apoio à redução do uso dos agrotóxicos na produção dos alimentos e monitoramento da qualidade das refeições oferecidas no âmbito do Programa de Saúde do Trabalhador.

Em 2016 não houve EM relacionadas ao M\&A. Em 2017, os conteúdos foram relativos ao monitoramento do PAA, do PNAE e da qualidade na produção de alimentos e no fornecimento de água. A necessidade da articulação entre SAN e políticas de igualdade racial também foi abordada.

A partir de 2018, não foram publicadas EM, sendo o período marcado pelo agravamento da crise econômica e institucional deflagrada pelo impeachment da presidente Dilma Rousseff em 2016, com cortes orçamentários em programas estratégicos para a SAN e limitação de investimentos em saúde e educação pelo mandato sucessor $^{45}$. Em 2019, a corrente neoliberal é reforçada com um novo mandato presencial e as EM deixaram de ser publicadas devido à extinção do CONSEA $^{15}$.

\section{Instrumentos e oportunidades para M\&A de SAN}

Durante a vigência do CONSEA, foram elaborados e publicados o I PLANSAN (2012-2015) e o II PLANSAN (2016-2019). Fruto da sistematização dos debates realizados nas CNSAN, pelo CONSEA e a CAISAN, os planos foram influenciados e subsidiados pelas discussões sobre indicadores de M\&A de SAN. Esses documentos reúnem dados referentes à situação de (in)segurança alimentar e nutricional e ao grau de implementação de programas e ações afeitos à SAN, permitindo identificar prioridades e propor articulações com as metas dos planos plurianuais ${ }^{46,49}$.

A comparação dos indicadores de cada plano mostra que o I PLANSAN utilizou um número maior de indicadores (57) do que o II PLANSAN (35). Essa diferença pode ser atribuída ao processo de refinamento das análises, que no segundo ocorreu de forma mais sistematizada, atendendo à demanda de subsidiar a construção das metas prioritárias e estratégicas para a $\mathrm{SAN}^{46,49}$.

Além das mudanças quantitativas, diferenças qualitativas são observadas nas dimensões em análise. No que se refere à Produção de alimentos (sete indicadores) e à Disponibilidade de alimentos (três indicadores), destaca-se sua desagregação no I PLANSAN, em contraposição ao II PLANSAN, em que essas dimensões foram agregadas em cinco indicadores. Não obstante a compreensão da interrelação entre ambas as dimensões, os indicadores elaborados no II PLANSAN apresentaram mais aspectos relacionados à produção do que voltados à disponibilidade.

Já a dimensão III (Renda e condições de vida), aparece, no II PLANSAN, com oito indicadores semelhantes aos 12 do I PLANSAN. Porém, os dados são desagregados por raça/cor, gênero, regiões urbanas e rurais ${ }^{46,49}$. Essa dimensão aparece combinada com a IV (Acesso à alimentação adequada e saudável, incluindo água) e a V (Saúde, nutrição e acesso a serviços relacionados) no II PLANSAN, com dois indicadores focando o estado nutricional de crianças indígenas e quilombolas, bem como a renda familiar com o PBF.

Outra diferença relevante diz respeito aos indicadores de consumo de frutas e hortaliças, além de produtos alimentícios e ingredientes culinários, como refrigerantes e sal. Isso sugere o impacto da nova classificação de alimentos ${ }^{50}$, incorporada à segunda edição do Guia alimentar para a população brasileira durante a publicação do II PLANSAN revisado ${ }^{49}$. Cabe ressaltar que o guia constitui uma meta do I PLANSAN para a promoção da alimentação adequada e saudável ${ }^{51}$. Da mesma forma, o indicador de monitoramento da qualidade dos alimentos está contemplado no II PLANSAN, com o reforço para análise de resíduos de agrotóxicos em alimentos vegetais tema bastante recorrente nas plenárias e discussões do CONSEA.

Os indicadores de Educação (dimensão VI) estão agregados a outros no II PLANSAN, notadamente àqueles relacionados ao acesso à alimentação adequada no ambiente escolar e nos serviços de saúde. Já o I PLANSAN apresenta quatro indicadores contidos nessa dimensão, que tratam de saneamento nas escolas de educação básica e anos de estudo. Em 2012, foi lançado o "Marco de referência de educação alimentar e nutricional para as políticas públicas", que in- 
cluiu nos objetivos e nas metas do I PLANSAN ações de incentivo à alimentação adequada e saudável, principalmente no ambiente escolar ${ }^{52}$.

No I PLANSAN, prevaleceram indicadores da dimensão V (Saúde, nutrição e acesso a serviços relacionados), associados ao monitoramento do estado nutricional, de carências nutricionais e da qualidade dos alimentos (resíduos de agrotóxicos e medicamentos veterinários). Igualmente, aqueles relativos ao monitoramento da aquisição de alimentos dentro e fora do domicílio (dimensão II - Disponibilidade de alimentos) e da extrema pobreza (dimensão III - Renda e condições de vida) também foram contemplados. No II PLANSAN, predominaram indicadores das dimensões III (extrema pobreza, com desagregação por regiões, gêneros, raça/cor) e IV (insegurança alimentar, despesas com alimentação, hábitos de consumo, consumo alimentar, qualidade e disponibilidade de alimentos, inclusive no ambiente escolar).

Os PLANSAN - elaborados no âmbito da CAISAN - dialogam com as EM publicadas pelo CONSEA, demonstrando a forte característica de articulação intersetorial promovida pela agenda de SAN desde a sua concepção, bem como pelo Decreto $\mathrm{n}^{\circ} 7.272 / 2010$, em que as dimensões de M\&A foram consolidadas ${ }^{5}$. Enquanto as EM analisam pontos favoráveis, desfavoráveis e de atenção para a concretização progressiva do DHAA e da SAN, os PLANSAN traduzem esses pontos em indicadores e metas de monitoramento, avaliação e proposição de ações e políticas relacionadas à SAN.

A perspectiva de M\&A empreendida no âmbito da SAN assume, na prática, diversos objetivos de avaliação descritos'. Mais do que isso, os processos e iniciativas de M\&A se retroalimentam, implementando ciclos avaliativos em detrimento de iniciativas estanques, que tendem a ser infrutíferas em relação aos resultados.

Segundo Champagne e colaboradores ${ }^{53}$, o objetivo derradeiro da avaliação é a melhoria social, por intermédio do aperfeiçoamento das instituições e da coerência das políticas e programas sociais. Indiscutivelmente, isso pode ser observado no período analisado.

\section{Considerações finais}

A importância de órgãos como o CONSEA e a CAISAN é evidenciada com a análise dos documentos publicados, cabendo destaque para a apresentação de propostas e conteúdos complementares entre si, alinhados aos contextos políticos e históricos de cada momento. As articulações intersetoriais - ou tentativas de - são a tônica da atuação desses órgãos, principalmente em relação ao M\&A de intervenções que impactam a SAN e o DHAA da população brasileira.

Com a extinção do CONSEA e outros conselhos-chave, documentos, conferências e demais formas de diálogo e articulação, sobretudo entre sociedade civil e governo, deixam de ser realizados. Isso implica a redução de espaços de participação social nos processos propositivos e decisórios, bem como a criação de barreiras para órgãos e entidades não governamentais na agenda de construção de políticas públicas. Da mesma forma, cria lacunas e reforça as antigas.

Embora parte das atribuições do CONSEA tenha sido absorvida pelo atual Ministério da Cidadania, no âmbito da Secretaria Especial do Desenvolvimento Social ${ }^{14}$, não foram observadas movimentações para a construção do III PLANSAN, gerando incerteza sobre a continuidade das ações e dos esforços que vinham sendo empreendidos.

Deve-se reconhecer que a abrangência do conceito de SAN impõe desafios complexos para a consolidação dos mecanismos de M\&A. Porém, foram observados desdobramentos positivos, com a valorização do conceito, nas ações de governo e nos marcos legais firmados ao longo do tempo.

Por fim, cabe destacar a importância da produção e valorização de trabalhos que evidenciem experiências e repercussões da atuação desses órgãos, seja analisando M\&A ou outras perspectivas, apesar de eventuais limitações de acesso a documentos e sites de domínio público.

\section{Colaboradores}

ASB Oliveira, JP Casemiro, AL Brandão e AMS Pinto participaram de todas as etapas da construção do artigo. 


\section{Referências}

1. Burlandy L. A atuação da sociedade civil na construção do campo da alimentação e nutrição no Brasil: elementos para reflexão. Cien Saude Colet 2011; 16(1):63-72.

2. Brasil. Decreto-lei no 11.346 , de 15 de setembro de 2006. Cria o Sistema Nacional de Segurança Alimentar e Nutricional (SISAN) com vistas em assegurar o direito humano à alimentação adequada e dá outras providências. Diário Oficial da União 2006; 15 set.

3. Contandriopoulos AP, Champagne F, Denis JL, PineaultR. A avaliação na área da saúde: conceitos e métodos. In: Hartz ZMA, organizadora. Avaliação em saúde: dos modelos conceituais à prática na análise da implantação de programas. Rio de Janeiro: Fiocruz; 1997. p. 29-49.

4. Santos SMC, Sampaio MFA. Contexto do planejamento e da avaliação da segurança alimentar e nutricional. In: Rocha C, Burlandy L, Magalhães R, organizadores. Segurança alimentar e nutricional: perspectivas, aprendizados e desafios para as políticas públicas. Rio de Janeiro: Fiocruz, 2013. p. 147-168.

5. Brasil. Decreto $\mathrm{n}^{\circ} 7272$, de 25 de agosto de 2010. Regulamenta a Lei no 11.346 , de 15 de setembro de 2006, que cria o Sistema Nacional de Segurança Alimentar e Nutricional - SISAN com vistas a assegurar o direito humano à alimentação adequada, institui a Política Nacional de Segurança Alimentar e Nutricional - PNSAN, estabelece os parâmetros para a elaboração do Plano Nacional de Segurança Alimentar e Nutricional, e dá outras providências. Diário Oficial da União 2010; 25 ago.

6. Cellard A. A análise documental. In: Nasser AC. A pesquisa qualitativa: enfoques epistemológicos e metodológicos. Petrópolis: Vozes; 2014. p. 295- 316.

7. Brasil. Conselho Nacional de Segurança Alimentar e Nutricional. Resolução no 1, de 25 de março de 2013. Diário Oficial da União 2013; 25 mar.

8. Bardin, L. Análise de conteúdo. São Paulo: Edições 70; 2011.

9. Figueiró AC, Frias PG, Navarro LM. Avaliação em saúde: conceitos básicos para a prática nas instituições. In: Samico I, Felisberto E, Figueiró AC, Frias PG, organizadores. Avaliação em saúde: bases conceituais e operacionais. Rio de Janeiro: Medbook; 2010. p. 1-14.

10. Brasil. Conselho Nacional de Segurança Alimentar e Nutricional. I Conferência Nacional de Segurança Alimentar. Brasília: CONSEA; 1995.

11. Burlandy L, Labra ME. Redes de política no combate à fome e à pobreza: a estratégia Comunidade Solidária no Brasil. Cien Saude Colet 2007; 12(6):1543-1552.

12. Burlandy L. A construção da política de segurança alimentar e nutricional no Brasil: estratégias e desafios para a promoção da intersetorialidade no âmbito federal de governo. Cien Saude Colet 2009; 14(3):851860 .

13. Brasil. Decreto no 6272, de 23 de novembro de 2007 . Dispõe sobre as competências, a composição e o funcionamento do Conselho Nacional de Segurança Alimentar e Nutricional - CONSEA. Diário Oficial da União 2007; 23 nov.
14. Brasil. Decreto $n^{\circ} 6273$, de 23 de novembro de 2007 . Cria, no âmbito do Sistema Nacional de Segurança Alimentar e Nutricional - SISAN, a Câmara Interministerial de Segurança Alimentar e Nutricional. Diário Oficial da União 2007; 23 nov.

15. Brasil. Medida Provisória no 870 , de $1^{\circ}$ de janeiro de 2019. Estabelece a organização básica dos órgãos da Presidência da República e dos Ministérios. Diário Oficial da União 2019; 1 jan.

16. Brasil. Veto $n^{\circ}$ 21/2019. Votação do dispositivo 21.19.004 - inciso XVI do "caput" do art. 24. Congresso Nacional 2019; 21 jun.

17. Brasil. Conselho Nacional de Segurança Alimentar e Nutricional. Documento Final do Encontro Nacional de Segurança Alimentar e Nutricional +2. Brasília: CONSEA; 2006.

18. Vaitsman J, Rodrigues RWS, Paes-Sousa R. O Sistema de Avaliação e Monitoramento das Políticas e Programas Sociais: a experiência do Ministério do Desenvolvimento Social e Combate à Fome do Brasil. Brasília: MDS; 2006.

19. Jannuzzi PM. Avaliação de programas sociais no Brasil: repensando práticas e metodologias das pesquisas avaliativas. Planejamento e Políticas Públicas 2011; 36:251-275.

20. Brasil. Presidência da República. Conselho Nacional de Segurança Alimentar e Nutricional. Construindo um sistema de monitoramento da realização progressiva do direito humano à alimentação adequada (DHAA), no contexto do Sistema Nacional da Segurança Alimentar e Nutricional (SISAN). Brasília; 2007

21. Brasil. Conselho Nacional de Segurança Alimentar e Nutricional. A segurança alimentar e nutricional e o direito humano à alimentação adequada no Brasil: indicadores e monitoramento da Constituição de 1988 aos dias atuais. Brasília: CONSEA; 2010.

22. Brasil. Presidência da República. Construção do Sistema e da Política Nacional de Segurança Alimentar e Nutricional: a experiência brasileira. Brasília; 2009.

23. Brasil. Ação Brasileira pela Nutrição e Direitos Humanos. Centro de Referência em Segurança Alimentar e Nutricional. Avanços e desafios na implementação do direito humano à alimentação adequada. Brasília: CONSEA; 2009.

24. Brasil. A exigibilidade do direito humano à alimentação adequada e o sistema Nacional de Segurança Alimentar e Nutricional - relatório final. Brasília; 2010.

25. Brasil. Câmara Interministerial de Segurança Alimentar e Nutricional. A exigibilidade do direito humano à alimentação adequada - ampliando a democracia no SISAN. Brasília; 2016.

26. Brasil. Presidência da República. Emenda Constitucional $n^{\circ} 64$. Altera o artigo $6^{\circ}$ da Constituição Federal, para introduzir a alimentação como direito social. Diário Oficial da União 2010; 4 fev.

27. Brasil. Câmara Interministerial de Segurança Alimentar e Nutricional. Estruturando o Sistema Nacional de Segurança Alimentar e Nutricional. Brasília: CAISAN; 2011. 
28. Mafra LAS, Naves FL. Gestão de políticas sociais: a importância das articulações institucionais e setoriais em programas de segurança alimentar e nutricional. Cadernos EBAPE. BR 2009; 7(1):34-49.

29. Vasconcellos ABPA, Moura LBA. Segurança alimentar e nutricional: uma análise da situação da descentralização de sua política pública nacional. Cad Saude Publica 2018; 34(2):e00206816.

30. Brasil. Câmara Interministerial de Segurança Alimentar e Nutricional. Estudo Técnico CAISAN: Mapeamento da insegurança alimentar e nutricional com foco na desnutrição a partir da análise do Cadastro Único, do Sistema Nacional de Vigilância Alimentar e Nutricional (SISVAN) e do Sistema de Informação da Atenção à Saúde Indígena (SIASI). Brasília: CAISAN; 2014.

31. Brasil. Ministério do Desenvolvimento Social e Combate à Fome (MDS). Secretaria Nacional de Segurança Alimentar e Nutricional. Secretaria de Avaliação e Gestão da Informação. Câmara Interministerial de Segurança Alimentar e Nutricional. MAPASAN 2014: mapeamento da segurança alimentar e nutricional. Brasília: MDS; 2015.

32. Brasil. Câmara Interministerial de Segurança Alimentar e Nutricional. Agroecologia e o direito humano à alimentação adequada. Brasília: CAISAN; 2012.

33. Brasil. Câmara Interministerial de Segurança Alimentar e Nutricional. Volatilidade dos preços internacionais e inflação de alimentos no Brasil: fatores determinantes e repercussões na segurança alimentar e nutricional. Brasília: CAISAN; 2013.

34. Brasil. Câmara Interministerial de Segurança Alimentar e Nutricional. Estratégia intersetorial de prevenção e controle da obesidade: recomendações para estados e municípios. Brasília: CAISAN; 2014.

35. Brasil. Câmara Interministerial de Segurança Alimentar e Nutricional. Compromissos do Brasil para a Década de Ação das Nações Unidas para a Nutrição (20162025). Brasília: CAISAN; 2016.

36. Brasil. Câmara Interministerial de Segurança Alimentar e Nutricional. Estratégia intersetorial para a redução de perdas e desperdício de alimentos no Brasil. Brasília: CAISAN; 2018.

37. Burity V, Franceschini T, Valente F, Recine E, Leão M, Carvalho MF. Direito humano à alimentação adequada no contexto da segurança alimentar e nutricional. Brasília: ABRANDH; 2010.

38. Oliveira JJ. A influência do Conselho Nacional de Segurança Alimentar e Nutricional no orçamento da União. In: Congresso CONSAD de Gestão Pública; 2017; Brasília. p. 1-16.

39. Vasconcelos FAG. Combate à fome no Brasil: uma análise histórica de Vargas a Lula. Rev Nutr 2005; 18(4):439-457.

40. Ramos MP, Lima LL. Avaliação de políticas sociais no Brasil: o caso do Programa Bolsa Família.In: Madeira LM, organizadora. Avaliação de políticas públicas. Porto Alegre: UFRGS; 2014. p. 64-82.

41. Brasil. Conselho Nacional de Segurança Alimentar e Nutricional. II Conferência Nacional de Segurança Alimentar e Nutricional. Olinda; 2004.

42. Santarelli M, Burity V; Basílio e Silva LN, Prates L, Rizzolo A, Rocha NC, Trabuco L. Da democratização ao golpe: avanços e retrocessos na garantia do direito $h u$ mano à alimentação e à nutrição adequadas no Brasil. Brasília: FIAN Brasil; 2017.
43. Brasil. Lei 11147, de 16 de junho de 2009. Dispõe sobre o atendimento da alimentação escolar e do Programa Dinheiro Direto na Escola aos alunos da educação básica; altera as Leis nos 10.880 , de 9 de junho de 2004, 11.273, de 6 de fevereiro de 2006, 11.507, de 20 de julho de 2007; revoga dispositivos da Medida Provisória no 2.178-36, de 24 de agosto de 2001, e a Lei no 8.913 , de 12 de julho de 1994; e dá outras providências. Diário Oficial da União 2009; 16 jun.

44. Brasil. Ministério da Cidadania. Secretaria Especial de Desenvolvimento Social. Apresentação [Internet]. [acesso 2020 Mar 21]. Disponível em: http://mds.gov. br/assuntos/brasil-sem-miseria/o-que-e

45. Vasconcelos FAG, Machado ML, Medeiros MAT, Neves JA, Recine E, Pasquim EM. Public policies of food and nutrition in Brazil: From Lula to Temer. Rev Nutr 2019; 32:e180161.

46. Brasil. Câmara Interministerial de Segurança Alimentar e Nutricional. I Plano Nacional de Segurança Alimentar e Nutricional (Revisado). Brasília: CAISAN; 2014.

47. Brasil. Conselho Nacional de Segurança Alimentar e Nutricional. Relatório final - mesa de controvérsias sobre transgênico. Brasília: CONSEA; 2013.

48. Brasil. Conselho Nacional de Segurança Alimentar e Nutricional. Mesa de controvérsias sobre impactos dos agrotóxicos na soberania e segurança alimentar e nutricional e no direito humano à alimentação adequada - relatório final. Brasília: CONSEA; 2014.

49. Brasil. Câmara Interministerial de Segurança Alimentar e Nutricional. II Plano Nacional de Segurança Alimentar e Nutricional. Brasília: CAISAN; 2018.

50. Monteiro CA, Cannon G, Levy RB, Moubarac JC, Louzada ML, Rauber F, Khandpur N, Cediel G, Neri D, Martinez-Steele E, Baraldi LG, Jaime PC. Ultra -processed foods: what they are and how to identify them. Public Health Nutr 2019; 22(5):936-941.

51. Brasil. Ministério de Saúde (MS). Secretaria de Atenção à Saúde. Departamento de Atenção Básica. Guia alimentar para a população brasileira. Brasília: MS; 2015.

52. Brasil. Ministério do Desenvolvimento Social e Combate à Fome (MDS). Secretaria Nacional de Segurança Alimentar e Nutricional. Marco de referência de educação alimentar e nutricional para as políticas públicas. Brasília: MDS; 2012.

53. Champagne F, Brousselle A, Hartz Z, Contandriopoulos AP, Denis JL. A análise de implantação. In: Brousselle A, organizador. Avaliação: conceitos e métodos. Rio de Janeiro: Fiocruz; 2011. p. 217-238.

Artigo apresentado em 23/07/2020
Aprovado em 14/12/2020
Versão final apresentada em 16/12/2020

Editores-chefes: Romeu Gomes, Antônio Augusto Moura da Silva 